\title{
Borboletas frugívoras (Lepidoptera: Nymphalidae) de uma área urbana (Área de Proteção Especial Manancial Cercadinho) em Belo Horizonte, Minas Gerais, Brasil
}

\author{
André Roberto Melo Silva ${ }^{1,3}$, Cíntia Oliveira de Castro ${ }^{1}$, Pedro Oliveira Mafia ${ }^{1}$, \\ Michelle Oliveira Campagnani Mendonça ${ }^{1}$, Tatiana Couto Castro Alves ${ }^{1} \&$ Marina do Vale Beirão $^{2}$ \\ ${ }^{1}$ Faculdade de Ciências Biológicas e da Saúde, Centro Universitário UNA, \\ Rua Guajajaras, 175, Centro, CEP 30180-100, Belo Horizonte, MG, Brasil \\ ${ }^{2}$ Instituto de Ciências Biológicas, Universidade Federal de Minas Gerais - UFMG, \\ Av. Antônio Carlos, 6627, Pampulha, CEP 31270-901, Belo Horizonte, MG, Brasil \\ ${ }^{3}$ Autor para correspondência: André Roberto Melo Silva, e-mail: andrerml@hotmail.com
}

SILVA, A.R.M., CASTRO, C.O., MAFIA, P.O., MENDONÇA, M.O.C., ALVES, T.C.C. \& BEIRÃO, M.V. Fruitfeeding butterflies (Lepidoptera: Nymphalidae) in an urban area (Area of Special Protection Manancial Cercadinho) in Belo Horizonte, Minas Gerais State, Brazil. Biota Neotrop. 12(3): http://www.biotaneotropica. org.br/v12n3/en/abstract?inventory+bn03312032012

\begin{abstract}
Aiming to increase the knowledge of fruit-feeding butterflies in an urban area in Minas Gerais State, a inventory was carried out in the Área de Proteção Especial Manancial Cercadinho, located in the suburban area of Belo Horizonte. Thirty baited traps were arranged in two habitats: 15 in a Cerrado area (Cerrado field) and 15 in a riparian forest. We recorded 1219 individuals belonging to 45 species of Nymphalidae. The rarefaction analyses didn't indicate difference in species richness between the riparian forest and Cerrado. The species accumulation curve did not show an asymptote. The four most abundant species belonged to Satyrinae. Our results about species richness in Cercadinho emphasize the need to protect this area, because it preserves $40 \%$ of the whole butterfly fauna estimated for the region, which could be managed as a source of colonization to other urban areas. Keywords: biodiversity, Cerrado, conservation, riparian forest, species richness.
\end{abstract}

SILVA, A.R.M., CASTRO, C.O., MAFIA, P.O., MENDONÇA, M.O.C., ALVES, T.C.C. \& BEIRÃO, M.V. Borboletas frugívoras (Lepidoptera: Nymphalidae) de uma área urbana (Área de Proteção Especial Manancial Cercadinho) em Belo Horizonte, Minas Gerais, Brasil. Biota Neotrop. 12(3): http://www. biotaneotropica.org.br/v12n3/pt/abstract?inventory+bn03112032012

Resumo: Com o objetivo de conhecer as borboletas frugívoras de uma área urbana em Minas Gerais, foi realizado um inventário na Área de Proteção Especial Manacial Cercadinho, localizada na periferia de Belo Horizonte. Foram instaladas 30 armadilhas em dois ambientes: 15 em uma área de Cerrado (campo Cerrado) e 15 na mata ciliar, durante o período de um ano. Foram coletados 1219 indivíduos pertencentes a 45 espécies da família Nymphalidae. A análise de rarefação não indicou diferença entre a riqueza de espécies da mata ciliar e do Cerrado. A curva acumulativa de ocorrência de espécies não resultou em uma assíntota. As quatro espécies mais abundantes pertencem à subfamília Satyrinae. Os resultados em relação à riqueza de espécies no Cercadinho apontam a importância da sua preservação, pois abriga $40 \%$ de toda a fauna de borboletas frugívoras estimadas para a região, podendo ser manejada como fonte de colonização de outras áreas urbanas.

Palavras-chave: biodiversidade, Cerrado, conservação, mata ciliar, riqueza de espécies. 


\section{Introdução}

A diversidade biológica não está homogeneamente distribuída pelo planeta, e o entendimento dos padrões de distribuição dos organismos é essencial para a tomada de decisões corretas visando a conservação (Ribeiro et al. 2008). Porém, o conhecimento sobre a biodiversidade ainda é escasso, principalmente de insetos em regiões tropicais, fator preocupante frente ao ritmo atual de destruição dos ecossistemas e extinção de espécies (Wilson 1997). Sem um conhecimento mínimo sobre quais organismos ocorrem em determinado local, é virtualmente impossível desenvolver qualquer projeto de conservação (Santos 2003), e este conhecimento é gerado através da realização de inventários de diversidade e posterior monitoramento.

Os insetos correspondem a quase $60 \%$ de todos os animais do planeta, mas têm sido pouco utilizados como espécies bandeira na conservação, mesmo sendo considerados excelentes indicadores biológicos (Freitas et al. 2003). Determinados grupos de insetos, como as borboletas, são importantes no monitoramento ambiental, pois respondem rapidamente a eventuais alterações, sendo fundamentais para a definição de conservação de áreas pequenas e hábitats fragmentados ou com longa história de influência antrópica (Freitas et al. 2003).

As borboletas frugívoras fazem parte da família Nymphalidae e estão distribuídas em quatro subfamílias: Satyrinae, Charaxinae, Biblidinae e Nymphalinae (Wahlberg et al. 2009), representando aproximadamente $20 \%$ da fauna de borboletas da região Neotropical (Lamas 2004). Estudos importantes já foram realizados envolvendo borboletas frugívoras, como por exemplo, os pioneiros de De Vries et al. $(1997,1999)$ e o mais atuais de Uehara-Prado et al. (2007) e Ribeiro et al. (2008).

Em Minas Gerais, estima-se que existam 1.600 espécies de borboletas, já que não existe uma lista disponível. Destas, 20 se encontram ameaçadas de extinção, sendo seis frugívoras (Casagrande et al. 1998). Apesar disso, existem apenas por volta de dez estudos sobre as comunidades de borboletas neste estado, sendo que os primeiros estudos foram de Brown Junior \& Mielke (1968), que publicaram uma lista para Belo Horizonte e Ebert (1969) que estudaram as borboletas de Poços de Caldas.

Além disso, nos últimos anos, aproximadamente oito estudos envolvendo também borboletas frugívoras foram realizados na região do cerrado brasileiro, local onde foi realizado o presente trabalho, como por exemplo, Pinheiro \& Ortiz (1992), que estudaram áreas de cerrado em Brasília, Fortunato \& Ruszczyk (1997), estudaram áreas de cerrado e matas de galeria na região central e periférica de Uberlândia, Soares et al. (1999), que estudaram áreas de cerrado ao longo da calha do Rio São Francisco, na região Noroeste de Minas Gerais e Pinheiro \& Emery (2006) que realizaram um levantamento das borboletas de uma área de proteção no Distrito Federal.

O objetivo do trabalho foi realizar um levantamento das espécies de borboletas frugívoras, comparando dois ambientes, cerrado e mata ciliar, da Área de Proteção Especial Manancial Cercadinho.

\section{Material e Métodos}

\section{1. Área de estudo}

A Área de Proteção Especial (APE) Manancial Cercadinho (19 58' 18,4" S e 43 54' 30" W), é administrada pela Companhia de Saneamento de Minas Gerais (COPASA-MG). Está localizada no sul do município de Belo Horizonte, em uma área urbana com 151 ha (Figura 1). Dentro da APE é realizada a captação de água que abastece alguns bairros da Zona Sul da capital mineira e parte da cidade de
Nova Lima, sendo o córrego do Cercadinho o principal contribuinte para a barragem de captação que integra o Sistema de Abastecimento Morro Redondo (Companhia... 2009).

A região apresenta duas estações, uma seca entre abril e setembro, e outra chuvosa entre outubro e março e o clima é o tropical de altitude. A APE Cercadinho é definida como uma área de transição entre o Cerrado e a Mata Atlântica, possuindo 40 ha de vegetação arbórea, incluindo a mata ciliar (Companhia... 2009). As fitofisionomias do cerrado presentes na área são cerradão e campo cerrado, este último formado por árvores e arbustos espaçados entre si, com um estrato contínuo de gramíneas e espécies herbáceas (Companhia... 2002). As coletas foram realizadas apenas no campo cerrado.

\section{Amostragem}

As amostragens foram realizadas mensalmente de outubro de 2006 a setembro de 2007 em duas áreas: cerrado (campo cerrado) e mata ciliar, com a metodologia de armadilhas atrativas para borboletas frugívoras.

As armadilhas consistem de um cilindro de tela fina com $90 \mathrm{~cm}$ de altura, fechado na extremidade superior e montado em uma plataforma de madeira com $35 \mathrm{~cm}$ de cada lado, com uma abertura de $3 \mathrm{~cm}$ para a entrada das borboletas (De Vries 1987). As borboletas entram pela abertura inferior, se alimentam na isca sobre a plataforma e, no momento de sair ficam presas no interior do cilindro (Freitas et al. 2003).

Foram utilizadas 30 armadilhas, sendo 15 na mata ciliar e 15 no cerrado. As mesmas foram dispostas em cinco unidades amostrais (UA), cada uma com três armadilhas distando 20 m entre si, e cada UA se distanciava $200 \mathrm{~m}$ uma da outra. As amostragens foram realizadas durante quatro dias, sendo que no primeiro dia as armadilhas eram instaladas e revisadas nos três dias subsequentes. A isca utilizada consistia de banana fermentada com caldo de cana, preparada 48 horas antes do início das amostragens.

Os espécimes capturados foram colocados em envelopes entomológicos numerados e conduzidos ao laboratório para posterior identificação. Dois indivíduos de cada espécie foram montados e a coleção está depositada no Laboratório de Zoologia do Centro Universitário UNA em Belo Horizonte. Indivíduos de fácil identificação em campo foram registrados, marcados e soltos.

A nomenclatura utilizada foi baseada em Lamas (2004). A divisão das subfamílias foi baseada em Wahlberg et al. (2009), considerando Morphini e Brassolini como tribos de Satyrinae.

\section{Análise dos dados}

Os dados foram analisados através da abundância e riqueza de espécies de borboletas frugívoras. Foi plotada a curva de suficiência amostral para verificar se a maior parte das espécies foram amostradas (Freitas et al. 2003). A constância das espécies foi calculada considerando: espécies presentes em mais de 50\% das coletas como constantes ou comuns, entre 25 e $50 \%$ (entre três e cinco coletas) acessórias e as coletadas em menos de $25 \%$ das amostragens (uma ou duas coletas) consideradas raras (Dajoz 1973). Foi realizada uma análise de rarefação baseada em indivíduos para comparar a riqueza de espécies entre cerrado e mata ciliar, através do programa Analytic Rarefaction 1.3.

\section{Resultados e Discussão}

Foram coletados 1219 indivíduos pertencentes a 45 espécies (Tabela 1). Brown Junior \& Mielke (1968), encontraram 50 espécies de borboletas frugívoras em Belo Horizonte e estimaram a presença de 107 espécies. Já Brown Junior \& Freitas (2000), registraram 80 espécies em uma localidade na Floresta Atlântica em Belo 


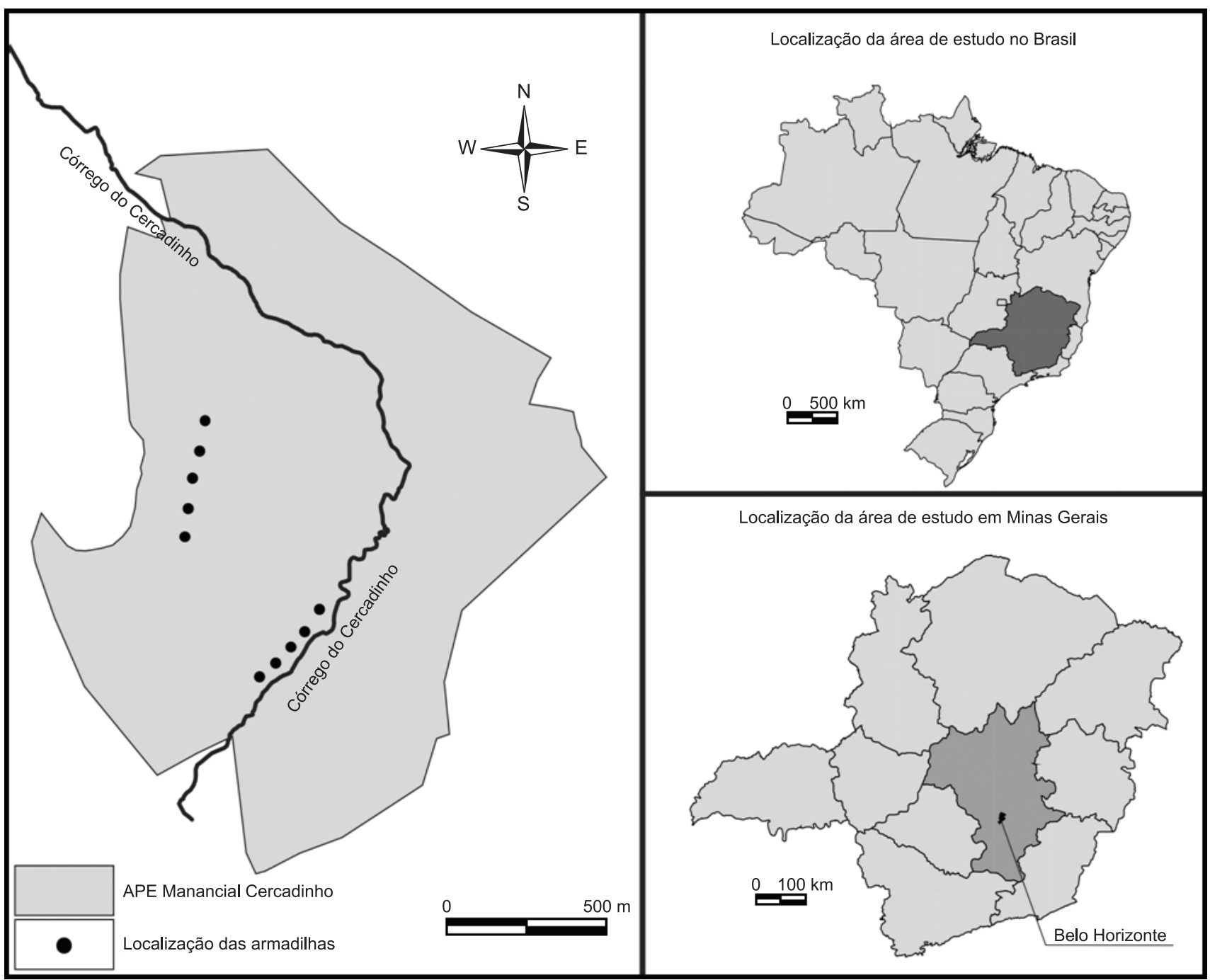

Figura 1. Localização da APE Cercadinho e das Unidades Amostrais (UA). Cada ponto representa uma unidade amostral com três armadilhas.

Figure 1. Location of APE Cercadinho and sampling points. Each point represents a sampling point with three traps.

Horizonte. A riqueza encontrada na APE Manancial Cercadinho equivale a $56 \%$ da fauna registrada por Brown Junior \& Freitas (2000) e a $42 \%$ do total estimado por Brown Junior \& Mielke (1968).

Vinte e três espécies pertencem à subfamília Satyrinae $(51,1 \%), 13$ a Biblidinae $(28,9 \%)$, seis a Charaxinae $(13,3 \%)$ e três a Nymphalinae $(6,7 \%)$. A subfamília com maior abundância foi Satyrinae com 955 indivíduos (78\%), seguida por Biblidinae (179 indivíduos; 15\%), Charaxinae (64 indivíduos; 5\%) e Nymphalinae (21 indivíduos; 2\%). A subfamília com maior riqueza de espécies na região Neotropical é Satyrinae, seguida por Biblidinae, Charaxinae, Brassolinae, Morphinae e Nymphalinae (Lamas 2004). O presente trabalho (se considerarmos Morphinae e Brassolinae como subfamílias) apresenta quase a mesma seqüência, apenas alterando a colocação dos dois últimos taxa, demonstrando que a comunidade de borboletas frugívoras em questão segue o padrão geral evidenciado para os Neotrópicos. Uma distribuição muito similar à do Cercadinho foi verificada por Pinheiro \& Ortiz (1992), no Jardim Botânico de Brasília, apenas com a diferença de Nymphalinae estar à frente de Brassolinae.

Comparações entre a riqueza obtida em diferentes trabalhos são difíceis de serem realizadas pela falta de padronização nos métodos de coleta e intensidade amostral. Entretanto, uma avaliação cuidadosa do número de espécies em diferentes áreas pode revelar informações interessantes. A riqueza de borboletas frugívoras encontradas no presente estudo foi maior que a registrada por Silva et al. (2007), estudando uma outra área urbana em Belo Horizonte (20 espécies), porém estes autores utilizaram apenas duas armadilhas (mais rede entomológica) ao longo de um ano. Fortunato \& Ruszczyk (1997), estudando áreas urbanas e extra urbanas em Uberlândia encontraram 36 espécies, utilizando 27 armadilhas ao longo de um ano e meio. Riqueza semelhante ao do presente trabalho foi encontrada por Pinheiro \& Ortiz (1992) em uma área de mata ciliar, cerrado e campo em Brasília, onde foram registradas 44 espécies, utilizando 14 armadilhas ao longo de um ano. AAPE Manancial Cercadinho apresentou maior riqueza em comparação às localidades acima citadas, sendo todas possuidoras de ambientes similares, além de 13 espécies exclusivas: Diaethria candrena, Epiphile hubneri, Archaeoprepona chalciope, Euptychoides castrensis, Godartiana muscosa, Moneuptychia itapeva, Pharneuptychia innocentia, Taygetomorpha celia, Taygetis rectifascea, Yphthimoides manasses, Yphthimoides renata, Caligo arisbe e Opoptera syme.

As quatro espécies mais abundantes pertencem à Satyrinae, sendo elas Y. manasses, Pharneuptychia sp.1, Taygetis laches e G. muscosa, representado respectivamente $21,6 \%, 8 \%, 7,5 \%$ e $7,1 \%$ do total de indivíduos. 
Tabela 1. Lista de espécies e abundância de borboletas frugívoras na mata ciliar e cerrado da Área de Proteção Especial Manancial Cercadinho, Belo Horizonte, Minas Gerais, Brasil, (outubro de 2006 e setembro de 2007).

Table 1. List of fruit-feeding butterflies species and abundance in the cerrado and riparian forest from the Área de Proteção Especial Manancial Cercadinho, Belo Horizonte, Minas Gerais State, Brazil, (October 2006 to September 2007).

\begin{tabular}{|c|c|c|}
\hline Subfamílias/tribos/espécies & Cerrado & Mata ciliar \\
\hline \multicolumn{3}{|l|}{ Biblidinae: Biblidini } \\
\hline Biblis hyperia nectanabis (Fruhstorfer, 1909) & 39 & 12 \\
\hline Callicore sorana (Godart, 1824) & 69 & - \\
\hline Callicore pygas thamyras (Ménétriés, 1857) & 3 & - \\
\hline Diaethria candrena (Godart, 1824) & 1 & - \\
\hline Diaethria clymena (Cramer, 1775)* & 7 & - \\
\hline Epiphile hubneri Hewitson, 1867 & - & 3 \\
\hline Eunica bechina (Hewitson, 1867)* & 1 & - \\
\hline Eunica cuvierii (Godart, 1819) & 1 & - \\
\hline Hamadryas amphinome amphinome (Linnaeus, 1767) & 8 & 4 \\
\hline Hamadryas epinome (Felder \& Felder, 1867) & - & 12 \\
\hline Hamadryas februa februa (Hübner, 1823)* & 10 & - \\
\hline Hamadryas feronia feronia (Linnaeus, 1758) & 4 & - \\
\hline Temenis laothoe meridionalis Ebert, 1965* & 5 & - \\
\hline \multicolumn{3}{|l|}{ Charaxinae: Anaeini } \\
\hline Fountainea ryphea (Cramer, 1775) & 24 & 13 \\
\hline Memphis moruus stheno (Prittwitz, 1865) & 7 & 8 \\
\hline Siderone galanthis (Cramer, 1775) & 3 & - \\
\hline Zaretis isidora (Cramer, 1777) & 5 & - \\
\hline \multicolumn{3}{|l|}{ Charaxinae: Preponini } \\
\hline Archaeoprepona chalciope (Hübner, 1823) & - & 1 \\
\hline Archaeoprepona demophon thalpius (Hübner, 1814) & 4 & 3 \\
\hline \multicolumn{3}{|l|}{ Nymphalinae: Coeini } \\
\hline Colobura dirce (Linneus, 1758)* & 1 & 13 \\
\hline Historis odius (Fabricius, 1775) & 4 & - \\
\hline Smyrna blomfildia (Fabricius, 1781)* & 1 & 2 \\
\hline \multicolumn{3}{|l|}{ Satyrinae: Satyrini } \\
\hline Euptychoides castrensis (Schaus, 1902) & 1 & 1 \\
\hline Godartiana muscosa (Butler, 1870) & 14 & 73 \\
\hline Moneuptychia itapeva Freitas, 2007 & 17 & - \\
\hline Paryphthimoides phronius (Godart, 1823) & 11 & 5 \\
\hline Paryphthimoides poltys (Prittwitz, 1865) & 5 & 2 \\
\hline Pharneuptychia innocentia (C. Felder e R. Felder, 1867) & 12 & 1 \\
\hline Pharneuptychia sp. 1 & 93 & 5 \\
\hline Pharneuptychia sp. 2 & 52 & 3 \\
\hline Taygetis laches (Fabricius, 1793)* & 24 & 67 \\
\hline Taygetis rectifascia Weymer, 1907 & 12 & 31 \\
\hline Taygetomorpha celia (Cramer, 1779) & - & 1 \\
\hline Yphthimoides celmis (Godart, 1824) & 31 & - \\
\hline Yphthimoides manasses (C. Felder e R. Felder, 1867) & 261 & 2 \\
\hline Yphthimoides ochracea (Butler, 1867) & 10 & 3 \\
\hline Yphthimoides renata $($ Stoll, 1780) & 62 & 16 \\
\hline Yphthimoides sp. 1 & 46 & 2 \\
\hline Yphthimoides sp. 2 & 5 & - \\
\hline \multicolumn{3}{|l|}{ Satyrinae: Morphini } \\
\hline Morpho helenor mielkei (Blandin, 2007) & 9 & 43 \\
\hline \multicolumn{3}{|l|}{ Satyrinae: Brassolini } \\
\hline Blepolenis batea batea (Hübner, 1821) & 1 & - \\
\hline Caligo arisbe Hübner, 1822 & - & 1 \\
\hline Eryphanis reevesii (Doubleday, 1849) & 3 & 3 \\
\hline Opoptera syme (Hübner, 1821) & 1 & 3 \\
\hline Opsiphanes invirae (Hübner, 1808)* & 20 & 3 \\
\hline Total & 883 & 336 \\
\hline
\end{tabular}




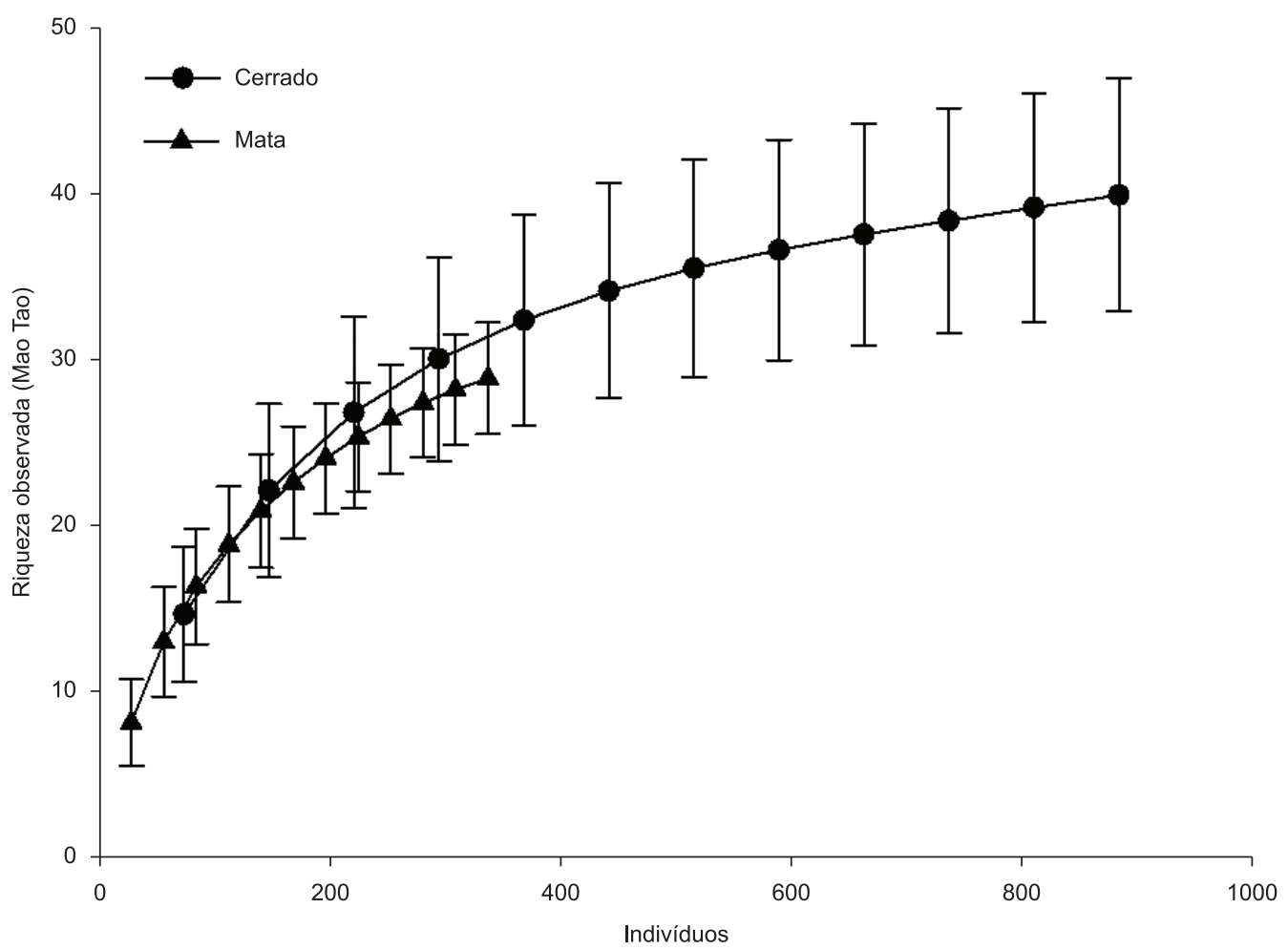

Figura 2. Curva de rarefação das borboletas frugívoras do cerrado e da mata ciliar, com o intervalo de confiança de $95 \%$.

Figure 2. Rarefaction curve of fruit-feeding butterflies of the Cerrado and riparian forest, with confidence interval (95\%).

Dezessete espécies foram constantes, 15 acessórias e 13 raras na área de estudo. Destas últimas, oito são singletons. Segundo Brown Junior \& Freitas (2000), embora a maioria dos grupos de borboletas possa sobreviver em fragmentos urbanos, muitas espécies são pouco representadas ou ausentes. Iserhard \& Romanowski (2004), sugerem que para a conservação de áreas de preservação e seu entorno, é aconselhável direcionar os esforços nas espécies de borboletas indicadoras ou raras, pois estes locais podem ser importantes para a conservação, por manter populações destas espécies. Das 45 espécies da APE Manancial Cercadinho, oito são consideradas comuns em ambientes antropizados por Brown Junior (1992) e Raimundo et al. (2003) (Tabela 1).

A curva de suficiência amostral não mostrou uma tendência nítida à estabilização após os 12 meses de coleta. Isto indica que apesar deste Parque possuir em torno de 50\% das espécies de borboletas frugívoras registradas para Belo Horizonte, o aumento do esforço amostral provavelmente acrescentaria mais espécies para a APE Manancial Cercadinho.

A análise de rarefação não mostrou diferença entre a riqueza de espécies da mata ciliar e cerrado (Figura 2). Entretanto, no cerrado foi encontrada maior riqueza e mais que o dobro da abundância de espécies do que na mata ciliar. Na mata ciliar, foram coletados 336 indivíduos de 29 espécies, e destas cinco foram exclusivas deste ambiente (E. hubneri, Hamadryas epinome, A. chalciope, T. celia e C. arisbe), representadas por 18 indivíduos. Já no cerrado, foram coletados 883 indivíduos pertencentes a 40 espécies, sendo 16 exclusivas, representadas por 167 indivíduos (Tabela 1).

Diferenças marcantes ocorrem na comunidade total de borboletas quando a floresta semidecídua dá espaço ao cerrado (Pinheiro \& Ortiz 1992, Brown Junior \& Freitas 2002), semelhante ao que ocorre no Cercadinho. A distribuição dos recursos de larvas e adultos poderia explicar os padrões de agrupamentos de espécies dentro de um mesmo fragmento, bem como características de temperatura, umidade e luminosidade (Ribeiro et al. 2008). No presente estudo, as duas fitofisionomias estudadas compreendem formações totalmente diferentes (mata e campo), influenciando a temperatura, umidade e luminosidade, fator que poderia explicar a diferença entre a abundância de borboletas frugívoras nestes locais. De acordo com Pinheiro \& Ortiz (1992) e Emery et al. (2006), a variedade de fitofisionomias do Cerrado provém uma grande heterogeneidade ambiental que proporciona uma variação espacial de distribuição de uma enorme variedade de borboletas, pois são encontrados microambientes propícios a uma ampla gama de espécies.

Isso evidencia a importante riqueza de borboletas frugívoras no cerrado, ecossistema historicamente pouco valorizado em relação à sua biodiversidade, ressaltando a importância de se amostrar várias fitofisionomias dentro de um mesmo bioma. Além disso, chama a atenção para a necessidade de um melhor conhecimento da fauna do Cerrado, considerado um hotspot mundial de diversidade (Myers et al. 2000).

A preservação de áreas verdes urbanas pode contribuir para a conservação da fauna de borboletas. Estes ambientes podem sustentar uma alta diversidade de espécies, por disponibilizar diferentes recursos e um local menos perturbado nas cidades, promovendo a melhoria do ecossistema urbano. Segundo Ruszczyk (1986) e Fortunato \& Ruszczyk (1997), para que haja uma riqueza considerável de borboletas em ambientes urbanos, é importante a preservação de fragmentos nativos no interior e periferia das cidades, pois são importantes como fonte de colonizadores potenciais do ambiente urbano.

Segundo Brown Junior \& Freitas (2002) e Ribeiro et al. (2008), para uma conservação efetiva de comunidades de borboletas em cidades de regiões tropicais, é necessário manter corredores de áreas verdes ao longo de ruas e avenidas, bem como reservas com água permanente (lagos ou córregos), vegetação nativa diversa e vegetação aberta. Sugere-se que tais locais sejam mais distantes 
dos aglomerados de edifícios dos grandes centros urbanos, e que se conectem com outras áreas verdes. A descrição acima se encaixa com as características da APE Manancial Cercadinho. Apesar de estar inserida em uma área urbana, localizada na periferia da cidade, também está próxima a outras áreas de preservação, como o Parque Estadual do Rola Moça, justificando possivelmente a alta riqueza de borboletas frugívoras registrada no presente trabalho.

Desta forma, o Cercadinho constitui-se numa área de preservação importante para a manutenção da riqueza de borboletas de Belo Horizonte, já que possui uma rica fauna de borboletas frugívoras, reforçando a importância de sua conservação.

\section{Agradecimentos}

Ao Centro Universitário UNA, à COPASA-MG, à bióloga Maria Aparecida Souza (COPASA-MG), ao professor Dr. André Victor Lucci Freitas (Universidade Estadual de Campinas, UNICAMP), pela identificação da maior parte dos espécimes, ao Dr. Márcio UeharaPrado e ao doutorando Luiz Dolabela Falcão pelo auxílio em algumas análises e a todos que ajudaram nas coletas.

\section{Referências Bibliográficas}

ANALYTIC RAREFACTION 1.3. http://www.uga.edu/strata/software/ AnRareReadme.html. (último acesso em 07/09/2011).

BROWN JUNIOR, K.S. 1992. Borboletas da Serra do Japi: diversidade, habitats, recursos alimentares e variação temporal. In História Natural da Serra do Japi: Ecologia e preservação de uma área florestal no Sudeste do Brasil. (L. Patrícia \& C. Morellato, eds.). FAPESP, Campinas, p.142-186.

BROWN JUNIOR, K.S. \& FREITAS, A.V.L. 2000. Diversidade de Lepidoptera em Santa Teresa, Espírito Santo. Bol. Mus. Biol. Mello Leitão (N. Ser.) 11-12:71-118.

BROWN JUNIOR, K.S. \& FREITAS, A.V.L. 2002. Butterfly communities of urban forest fragments in Campinas, São Paulo, Brazil: Structure, instability, environmental correlates, and conservation. J. Insect Conserv. 6(4):217-231. http://dx.doi.org/10.1023/A:1024462523826

BROWN JUNIOR, K.S. \& MIELKE, O.H.H. 1968. Lepidoptera of the Central Brazil Plateau. III. Partial list for the Belo Horizonte area, showing the character of the southeastern "Blend Zone". J. Lepid. Soc. 22(3):147-157.

CASAGRANDE, M.M., MIELKE, O.H.H. \& BROWN JUNIOR, K.S. 1998 Borboletas (LEPIDOPTERA) ameaçadas de extinção em Minas Gerais, Brasil. Rev. Bras. Zool. 15(1): 241-259. http://dx.doi.org/10.1590/S010181751998000100021

COMPANHIA DE SANEAMENTO DE MINAS GERAIS - COPASA. 2002. Estudos, diagnóstico, proposições e recomendações para a preservação da Área de Proteção do Manancial do Cercadinho. COPASA. Relatório Técnico.

COMPANHIA DE SANEAMENTO DE MINAS GERAIS - Copasa. 2009. Reservas Ambientais. COPASA. http://www.copasa.com.br/cgi/cgilua. exe/sys/start.htm?infoid=96\&sid=157\&tpl=section $\% 2$ Ehtm (último acesso em 15/11/2009).

DAJOZ, R. 1973. Ecologia Geral. Vozes, Petrópolis, 471p.

DE VRIES, P.J. 1987. The butterflies of Costa Rica and their natural history. Papilionidae, Pieridae, Nymphalidae. Princeton University Press, Princeton, 327p.

DE VRIES, P.J., MURRAY, D. \& LANDE, R. 1997. Species diversity in vertical, horizontal, and temporal dimensions of a fruit-feeding butterfly community in an Ecuadorian rainforest. Biol. J. Linn. Soc. 62:343-364. http://dx.doi.org/10.1111/j.1095-8312.1997.tb01630.x

DE VRIES, P.J., WALLA, T.R. \& GREENEY, H.F. 1999. Species diversity in spatial and temporal dimensions of a fruit-feeding butterfly community from two Ecuadorian rainforest. Biol. J. Linn. Soc. 68:333-353.

EBERT, H. 1969. On the frequency of butterflies in eastern Brazil, with a list of butterfly fauna of Poços de Caldas, Minas Gerais. J. Lepid. Soc. 23 (suplem. 3):1-48.
EMERY, E.O., BROWN JUNIOR, K.S. \& PINHEIRO, C.E.G. 2006. As borboletas (Lepidoptera, Papilionoidea) do Distrito Federal, Brasil. Rev. Bras. Entomol. 50(1):85-92.

FORTUNATO, L. \& RUSZCZYK, A. 1997. Comunidades de Lepidópteros frugívoros em áreas verdes urbanas e extra-urbanas de Uberlândia, MG. Rev. Brasil. Biol. 57(1):79-87.

FREITAS, A.V.L., FRANCINI, R.B. \& BROWN JUNIOR, K.S. 2003. Insetos como indicadores ambientais. In Métodos de estudos em biologia e manejo da vida silvestre. (L. Cullen Junior, R. Rudran \& C. ValladaresPadua, eds.). Editora da UFPR, Curitiba, p.125-151.

ISERHARD, C.A. \& ROMANOWSKI, H.P. 2004. Lista de espécies de borboletas (Lepidoptera, Papilionoidea e Hesperioidea) da região do vale do rio Maquiné, Rio Grande do Sul, Brasil. Rev. Bras. Zool. 21(3):649-662. http://dx.doi.org/10.1590/S0101-81752004000300027

LAMAS, G. 2004. Atlas of Neotropical Lepidóptera: Checklist. Part 4A - Hesperioidea e Papilionoidea. Scientific Publishers, Gainesville, 439p.

MYERS, N., MITTERMEIER, R.A., MITTERMEIER, C.G., FONSECA, G.A.B. \& KENT, J. 2000. Biodiversity hotspots for conservation priorities. Nature, 3:853-858.

PINHEIRO, C.E.G. \& EMERY, E.O. 2006. As borboletas (Lepidoptera: Papilionoidea e Hesperioidea) da Área de Proteção Ambiental do Gama e Cabeça de Veado (Distrito Federal, Brasil). Biota Neotrop. 6(3):1-15. http:// www.biotaneotropica.org.br/v6n3/pt/abstract?article+bn01506032006 (último acesso em 22/07/2011).

PINHEIRO, C.E.G. \& ORTIZ, J.V.C. 1992. Communities of FruitFeeding Butterflies along a vegetation gradiente in Central Brazil. J. Biogeog. 19:505-511. http://dx.doi.org/10.2307/2845769

RAIMUNDO, R.L.G., FREITAS, A.V.L., COSTA, R.N.S., OLIVEIRA, J.B.F., LIMA, A.F., MELO, A.B. \& BROWN JUNIOR, K.S. 2003. Manual de monitoramento ambiental usando borboletas e libélulas: Reserva extrativista do Alto Juruá, Marechal Thaumaturgo, Acre. UNICAMP, Campinas, 36p.

RIBEIRO, D.B., PRADO, P.I., BROWN JUNIOR, K.S. \& FREITAS, A.V.L. 2008. Additive partitioning of butterfly diversity in a fragment landscape: importance of scale and implications for conservation. Diversity Distrib. 14(6):961-968. http://dx.doi.org/10.1111/j.14724642.2008.00505.x

RUSZCZYK, A. 1986. Ecologia urbana de borboletas, I. O gradiente de urbanização e a fauna de Porto Alegre RS. Rev. Brasil. Biol. 46(4):675-688.

SANTOS, A.J. 2003. Estimativas de riqueza em espécies. In Métodos de estudos em biologia e manejo da vida silvestre. (L. Cullen Junior, R. Rudran \& C. Valladares-Padua, eds.). Editora da UFPR, Curitiba, p.19-42.

SILVA, A.R.M., LANDA, G.G. \& VITALINO, R.F. 2007. Borboletas (Lepidoptera) de um fragmento de mata urbano em Minas Gerais, Brasil. Lundiana 8(2):137-142.

SOARES, A., JÚLIO, C.E.A., CAETANO, C.A., TANGERINI, N., FARIA, A.C. \& COSTA, J.E. 1999. Lista da lepidopterofauna diurna da região da calha do Rio São Francisco, estado de Minas Gerais, municípios de Itacarambi, Jaíba, Manga e Matias Cardoso. Bol. Mus. Nac. N. S. Zool. 402:1-12.

UEHARA-PRADO, M., BROWN JUNIOR, K.S. \& FREITAS, A.V.L. 2007. Species richness, composition and abundance of fruit-feeding butterflies in the Brazilian Atlantic Forest: comparison between a fragmented and a continuous landscape. Global Ecol. Biogeogr. 16: 43-54. http://dx.doi. org/10.1111/j.1466-8238.2006.00267.x

WAHLBERG, N., LENEVEU, J., KODANDARAMAIAH, U., PEÑA, C., NYLIN, S., FREITAS, A.V.L \& BROWER, A.V.Z. 2009. Nymphalid butter lies diversify following near demise at the Cretaceous/Tertiary boundary. Proc R Soc Biol Sci Ser B 276: 4295-4302

WILSON, E.O. 1997. A situação atual da diversidade biológica. In Biodiversidade. (E.O. Wilson \& Peter, F.M. eds). Editora Nova Fronteira, Rio de Janeiro, p.3-24. 